\title{
Zika virus outbreaks in Asia and South America
}

A

$\mathrm{n}$ emerging mosquito-borne virus with outbreaks in Southeast Asia and South America is raising concern because of a spike in microcephaly in newborns in affected areas. Zika virus is also being linked to a rise in Guillain-Barré syndrome. But more data are needed before these outcomes can be definitively linked to the virus, say experts. In the meantime, Canadian physicians may see cases in patients arriving or returning from these regions.

A case of Zika virus has already been found in a traveller coming back to Canada from Thailand, said Dr. Bonnie Meatherall, an infectious disease physician with the University of Calgary, who treated the patient in early 2013.

The symptoms were similar to those in people infected with dengue virus, another mosquito-borne virus, said Meatherall. The Canadian patient recovered fully from the mild disease, which is rarely fatal. But public health authorities in affected countries are now investigating troubling possible aftereffects.

"It was thought to cause fever for a few days, muscle soreness, rash, and then settle, but in Brazil they noticed an increased rate of microcephaly," said Dr. Jonathan Gubbay, a medical microbiologist with Public Health Ontario. "Timing-wise, it occurred at the same time as the virus: March 2014."

The Pan American Health Organization issued an epidemiological alert on Dec. 1, 2015, after finding a 20-fold increase in the rate of microcephaly in Brazil this year. There, the virus has been found in the amniotic fluid of two pregnant women whose fetuses had the serious neurological malformation. Public health authorities in French Polynesia have also found an unusual increase in nervous system malformations in infants born in 2014-15, during a Zika virus outbreak there. There has also been an increase in cases of Guillain-Barré syndrome in people of all ages in both regions.

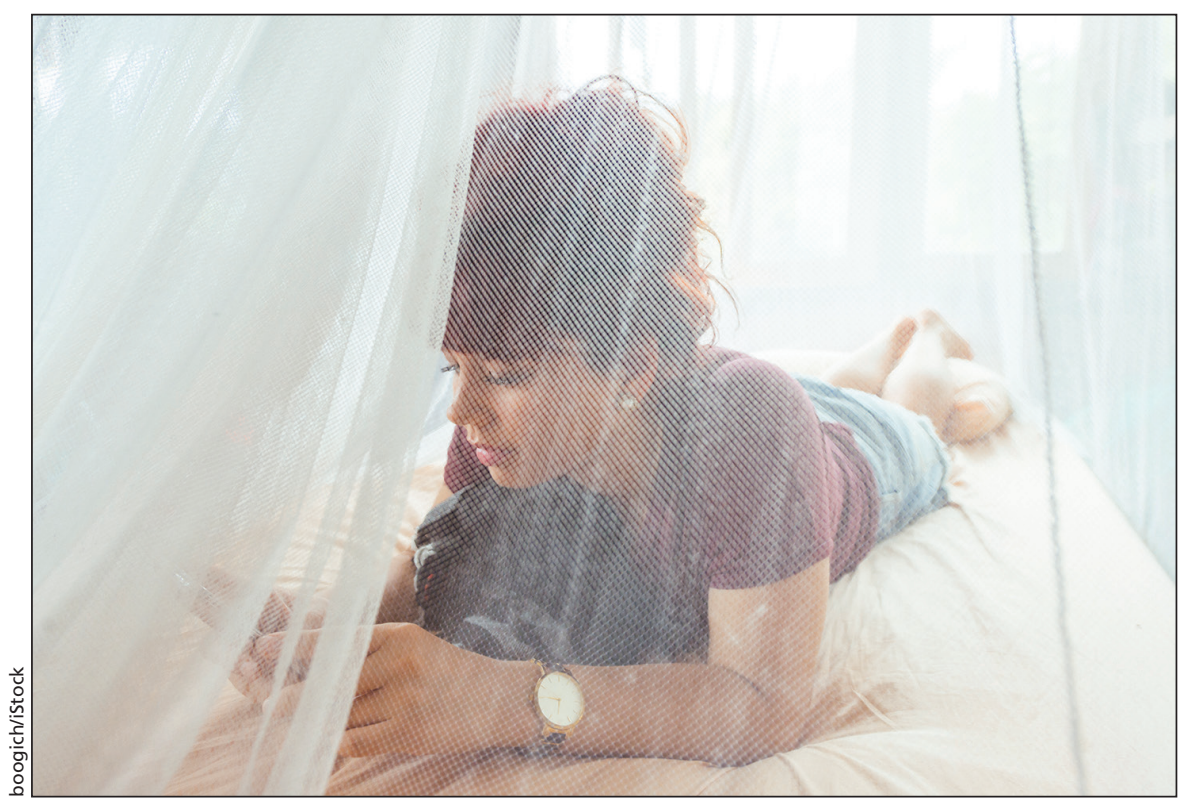

Travellers to areas where Zika is common should protect themselves from mosquitos.

"There's a strong signal of an association," said Gubbay, "but not enough to say there's a definite association."

Meatherall said not enough is known about the viruses' effects. "The microcephaly is a very interesting correlation that needs a lot more work. If this does end up being as a result of Zika virus, that's important to know." She believes it is important to distinguish imported cases of Zika virus from other viruses, as the advice to patients may differ, but points out that diagnosis is difficult. Symptoms of Zika virus infection are similar not only to Dengue, which has been found in many Canadian patients, but also to illness caused by infection with another mosquito-borne virus, chikungunya. "All three viruses are starting to circulate in the same area and have the same disease presentations."

In the Canadian case, initial blood tests indicated dengue virus infection, but more sensitive polymerase chain reaction tests were negative, prompting further testing at the US Centers for Disease Control and Canada's National Microbiology Laboratory to identify the virus.
Gubbay said that for most patients, it is not clinically important which virus they have, "but it will be important for us to monitor whether we have imported cases coming in." He said he would try to diagnose the exact virus in pregnant women, but not necessarily in all patients.

Both experts say it is unlikely that the virus would start to circulate in Canada. The mosquito species spreading Zika virus are not found here, although Canada has other mosquito species in the same Aedes genus.

For travellers to affected countries, Gubbay emphasizes prevention. "Wherever this virus exists, there are other diseases that are potentially more important to protect ourselves from," including malaria. "It is important for people to remember to take basic mosquito protection action" such as insect repellent, clothing, screens on windows and mosquito nets on beds, as well as emptying standing water that can create breeding sites. "And any patients who come back with a fever should make sure they see their doctor." - Carolyn Brown, Ottawa, Ont. 